\title{
Radial Interference Contrast in in-situ SEM Observation of Metal Oxide Semiconductor Film Crystallization
}

\author{
Kunji Shigeto $^{1}$, Takio Kizu ${ }^{2}$, Kazuhito Tsukagoshi ${ }^{2}$ and Toshihide Nabatame ${ }^{2}$ \\ 1. Hitachi High-Technologies Corporation, Kawasaki, Japan. \\ 2. NIMS MANA, Tsukuba, Japan.
}

Thin-Film Transistors (TFTs) based on transparent amorphous-oxide semiconducting films are in strong demand for high-speed switching operations in next-generation flat panel displays. Amorphous InGaZnO (a-InGaZnO) TFTs have been recognized as the most promising candidate at the moment [1]. Although the a-InGaZnO TFT has been partially used in commercial displays, there is controversy on the stability issues caused by oxygen vacancies (Vo) [2], which generate excess charge carriers in the films. As an index to seek an effective Vo suppressant, the bond dissociation energy between additive atoms and oxygen is found to be a reasonable parameter for selecting the additive into the oxide film matrix [3]. Among the additives with high bond dissociation energy, Si or W effectively suppresses the instability issue of InOx-based TFTs [4]. To better understand the role of additive atoms in the oxide film, here, we characterized crystallization dynamics of indium silicon oxide (InSiO) and indium tungsten oxide (InWO) by in-situ SEM observation. Results show that unique backscattered electron (BSE) images were obtained only in InSiO, possibly due to electron interference in the crystal plane.

Amorphous films of with a thickness of 30nm were prepared on sapphire substrates by means of DC magnetron sputtering at room temperature. Sputtering target of $\mathrm{InSiO}$ was composed of $97 \mathrm{wt} . \% \mathrm{In}_{2} \mathrm{O}_{3}$ with 3 wt. $\% \mathrm{SiO}_{2}$. The InWO target was composed of 90 wt. $\% \mathrm{In}_{2} \mathrm{O}_{3}$ with 10 wt. $\% \mathrm{WO}_{3}$. Corresponding atomic ratio of $\mathrm{Si}$ and $\mathrm{W}$ are 2.6 and 2.4 respectively, thus indicating comparable additive ratios in the two films. In-situ SEM observations were performed utilizing the combination of heating sub-stage Gatan Murano and Schottky SEM Hitachi SU5000. This combined system allows us to obtain images of crystallization dynamically along a precisely fixed observation area.

Crystallization of InWO started at $260^{\circ} \mathrm{C}$, and imaging with the BSE signal (Fig. 1(A)) showed plane contrast in each crystal. Uniform contrast (dark or bright spots) in each spot indicates crystallization along the gray background area of the amorphous film. While maintaining the temperature at $260^{\circ} \mathrm{C}$, each uniform contrast spot gradually increased in size as well as the density. Finally the crystals covered the entire surface of the film (Fig. 1(B)). The results rather show usual crystallization behavior frequently observed in the amorphous thin film. We also prepared a reference InWO film which contains 1.2 at. $\% \mathrm{~W}$ for a comparison with the result of 2.4 at. $\% \mathrm{~W}$. Although crystallization temperature was slightly lowered to $200^{\circ} \mathrm{C}$, the obtained SEM images were similar as the images shown in Figure 1.

In contrast to the InWO, the InSiO film showed pronounced differences. The InSiO film partially crystallized at $350^{\circ} \mathrm{C}$. Observations by $\mathrm{BSE}$ signal of $\mathrm{InSiO}$ were continuously acquired during the crystallization phase (Fig. 2(A)). The gray background area corresponds to the initial state of the amorphous film and the area with ordered contrast corresponds to the crystallized one, showing radial crystal contrasted patterns in the micron scale. Keeping the temperature of $350^{\circ} \mathrm{C}$, each crystal became larger and further developed crystallization spots in the film. Eventually the entire surface was covered by the radial-pattern crystals (Fig. 2(B)) 
The radial contrast in the BSE image (Fig. 2) can be clearly and reproducibly obtained in the InSiO films. According to the general understanding of BSE images, contrast differences suggests multiple domain and /or grain caused by mis-orientation of the crystals. Further observation by Electron BackScatter Diffraction (EBSD) analysis for InSiO, shows no existence of grain and /or domain boundary corresponding to the radial contrast of the BSE image. It was found that EBSD resolves single crystal in each spot in Figure 2(A).

According to our understanding, it is most likely the radial pattern of the crystal could be electron interference patterning in the crystals. We would like to discuss more detail of the observed unique contrast mechanism by EM observation, and crystallization dynamic effects obtained by our newly developed our combined in-situ SEM system.

References:

[1] K Nomura et al, Nature 432 (2004) 488.

[2] T Kamiya et al, NPG Asia Mater. 2 (2010) 15.

[3] S Aikawa et al, App. Phys. Lett. 103 (2013) 172105.

[4] N Mitoma et al, App. Phys. Lett. 106 (2015) 042106.
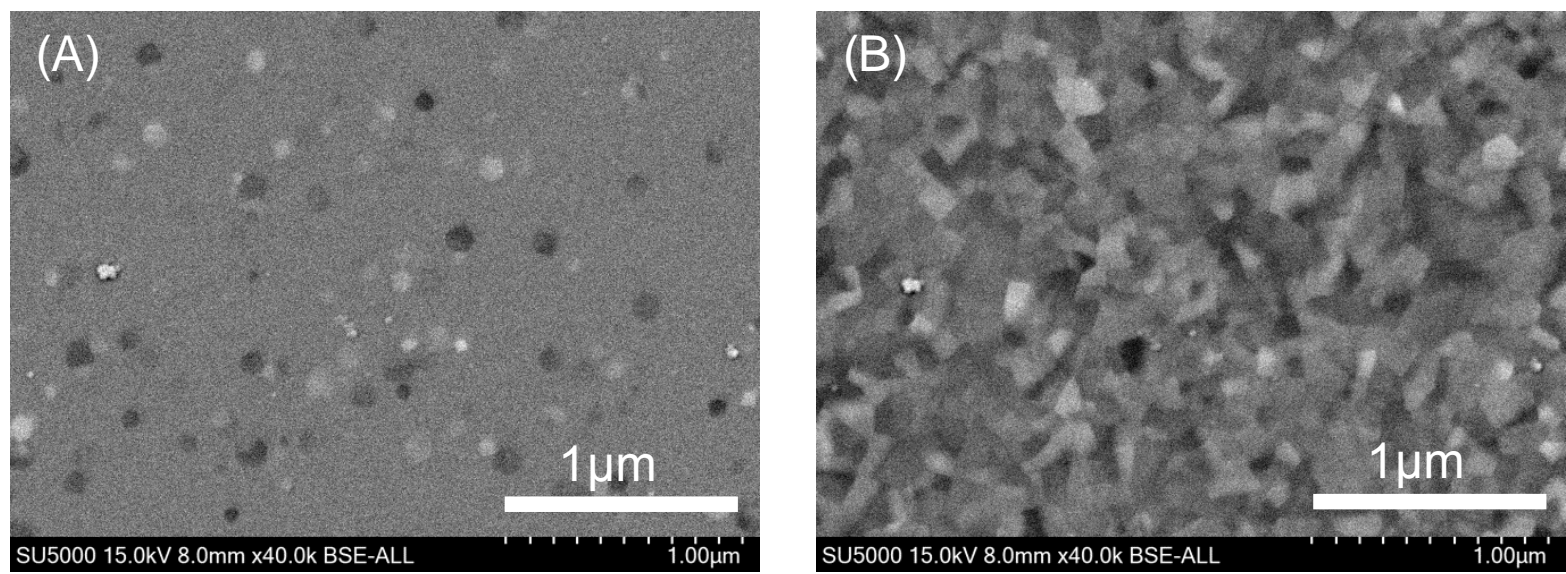

Figure 1. BSE images of InWO film. (A) In the middle of crystallization process of amorphous film at 260 C. (B) Final state of crystallization. Both images were obtained at the same field of view.
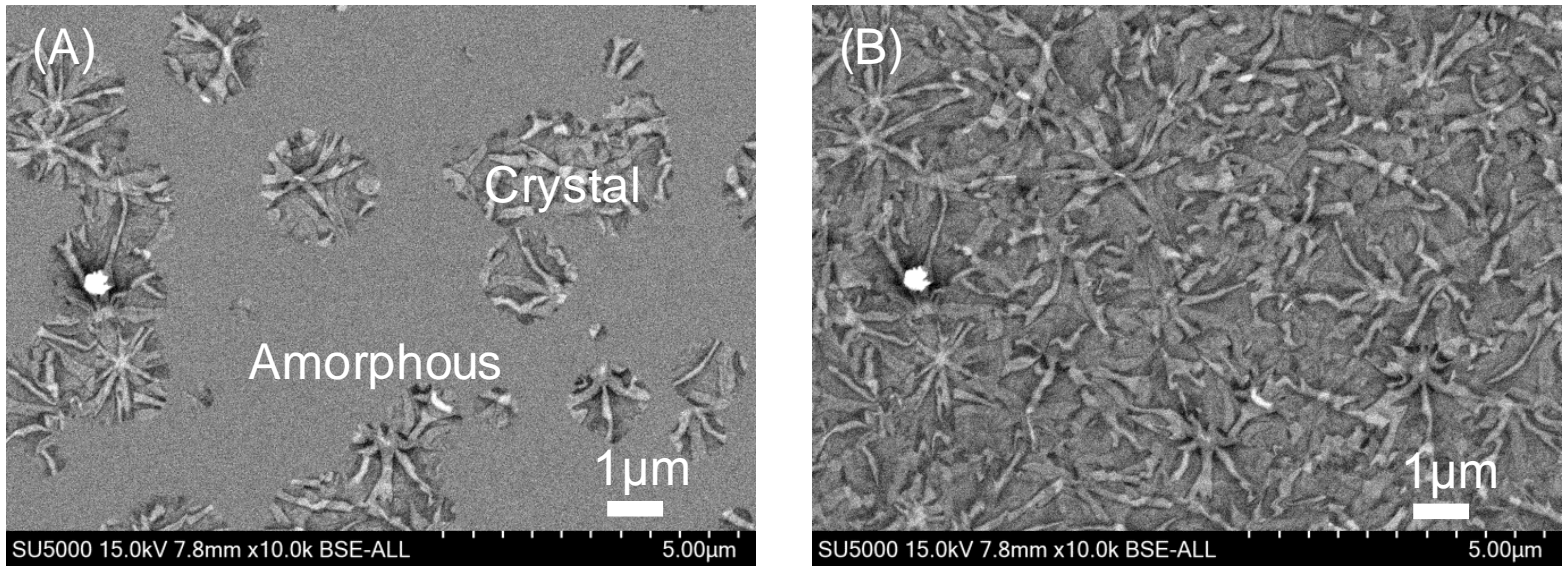

Figure 2. Unique BSE images of InSiO film. (A) In the middle of crystallization process of amorphous film at 350 C. (B) Final state of crystallization. Both images were obtained at the same field of view. 